\title{
Hot Pressed Ceramic Veneers for Diastemas Closure
}

\author{
Zeineb Riahii ${ }^{*}$, Anissa Ben Moussa ${ }^{2}$, Sawssen Belaid ${ }^{3}$, Belhassen Harzallah ${ }^{4}$ and Mounir Cherif ${ }^{5}$ \\ ${ }^{1} D D S$, Fixed Prosthodontic Department, Dental University of Monastir, Tunisia \\ ${ }^{2}$ Professor, Fixed Prosthodontic Department, Dental University of Monastir, Tunisia \\ ${ }^{3}$ Laboratory Technician, Fixed Prosthodontic Department, Dental University of Monastir, Tunisia \\ ${ }^{4}$ Director of the Resarch laboratory, Fixed Prosthodontic Department, Dental University of Monastir, Tunisia \\ ${ }^{5}$ Head of the Department, Fixed Prosthodontic Department, Dental University of Monastir, Tunisia \\ *Corresponding Author: Zeineb Riahi, DDS, Fixed Prosthodontic Department, Dental University of Monastir, Tunisia.
}

Received: October 25, 2019; Published: November 11, 2019

DOI: $10.31080 /$ ASDS.2019.03.0698

\begin{abstract}
Porcelain veneers are a common aesthetic treatment strategy, particularly for anterior teeth rehabilitation. Many studies have been conducted to show the important survival rate of those prosthetic pieces, which is comparable to that of metal ceramic restorations (Pjetursson., et al. 2007) [5].

Different dental ceramic systems are available to provide aesthetic solutions. The choice would be based on several parameters such as: the shade of the final restoration, but also the prepared teeth, the functional loading: whether it's on the anterior or the posterior region, the occlusal context...and other technical considerations related to the veneers preparation, manufacturing and bonding [10].

Ceramic materials can be classified based on their sinterization temperature, composition or manufacturing technique. According to their manufacturing technique ceramics can be classified into:

- $\quad$ Powde/liquid building.

- $\quad$ Slip casting.

- Hot pressed ceramic.

- $\quad$ CAD/CAM [4].

The hot pressed ceramic fabrication technique was introduced in 1980s based on waxed restorations built up by the technician before proceeding to the final prostheses manufacturing.

Those ceramics contain a high amount of glass offering an important aesthetic incomeof the restorations. Thus, this ceramic material became one the most used to fabricate aesthetic veneers [10].

Keywords: Ceramic Veneers; Hot Pressing Technique; Glass Based Ceramics; Laminate Veneers
\end{abstract}

\section{Introduction}

Aesthetic and conservative dentistry are common terms in our practice nowadays. Therefore studies and continued development of dental materials aim to reach the most aesthetic and conservative treatment option. To reach success in prosthetic rehabilitation, this one should be functional, aesthetically satisfactory and biologically compatible. One of the most important parameters to be considered to reach this aim is the material choice. 
Based their revolutionary aesthetic income, ceramics should be first materials nominated for an aesthetic rehabilitation.

Dental ceramics are proven to be the most aesthetic dental materials used for indirect restorations. However, this income is changeable from one system to another, making the choice a hard step to make.

The second parameter to be considered is the prosthetic option chosen: bridge, crown, inlay, veneer... [6].

Based on their longevity, conservative nature, biocompatibility and aesthetic income, veneers are one of the most indicated treatment modalities since their introduction. Initially indicated for slightly discoloured teeth, they do have now an indication average, widely covering anterior unaesthetic problems occupying patients. Although more conservative techniques are showing up such as bleaching and microabrasion, it has not led to decrease veneers indications, on the contrary, indicating veneers kept increasing thanks to materials development and new bonding techniques [11].

This paper will be a case report of ceramic dental veneers using the hot pressing technique, showing their pros and cons andt the steps of manufacturing.

\section{Case presentation}

A 40-year-old-male-patient presented to our department with a chief complaint "unpleasnt aesthetics due the presence of spaces between his anterior teeth".

The clinical examination showed a satisfactory oral hygiene. Several distemas between the front teeth were noticed; spaces of more than $0.5 \mathrm{~mm}$ between the canine, the lateral incisor and the central one on both sides of the maxilla. Slight mesial rotations of both centrals were also observed. The patient has Angle's class I occlusion. The patient refused the orthodontic treatment and asked for a non invasive prosthetic treatment option. Therefore, the decision was to make 6 ceramic veneers from canine to canine in bot sides of the maxilla. Since no color problems were observed, we opted for E max press system.

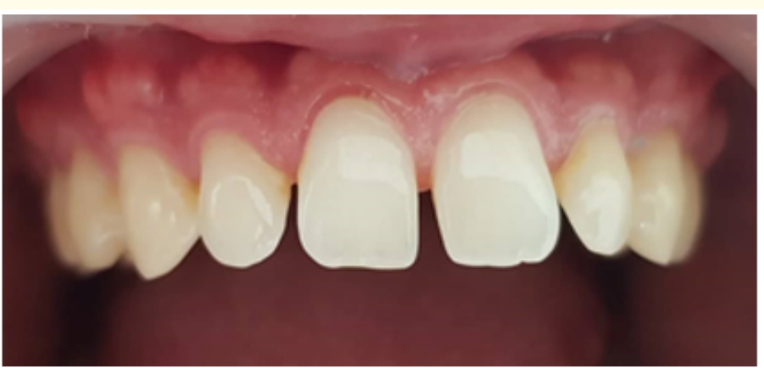

Figure 1: Intraoral front view at the first session.

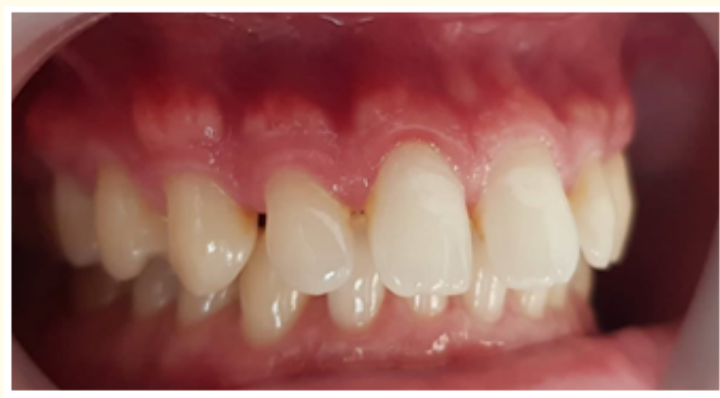

Figure 2: Intraoral side view at the first session.

The diagnostic cast was then waxed, and used to fabricate a silicone index for mock up and provisionals. After that, teeth preparations were made with minimal reductions particularly on mesial sides of both central incisors, since their slight rotation. To guarantee an optimal bonding of the veneers, the preparation should be located in the enamel. The reduction mustn't exceed $0.6 \mathrm{~mm}$ in the labial area and $0.7 \mathrm{~mm}$ in the incisal edge. This managed space would be enough to make a resistant veneer with the pressed technique (Figure 3).

After master impression was taken, the fabrication of the ceramic veneers was soon started in the dental laboratory. 


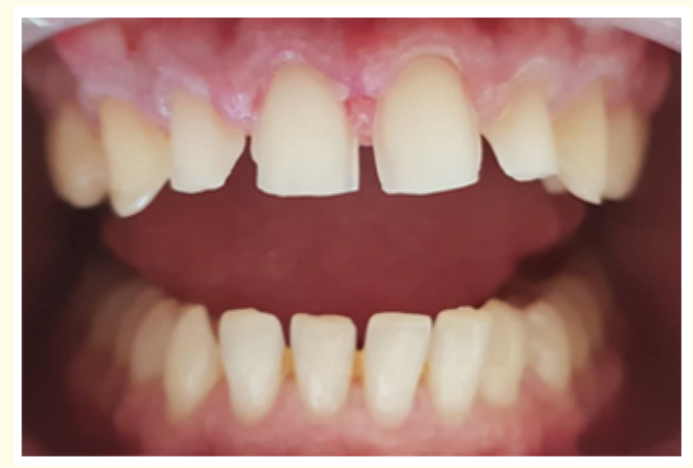

Figure 3: Intraoral view of teeth preparations.

\section{Laboratory steps for veneers fabrication}

The working model is fabricated and a die spacer is applied up to $1 \mathrm{~mm}$ from the margin. The die is waxed up: an organic wax or a resin that burns out without leaving any residues is used. The staining technique was used respecting the final wall thicknesses and the framework design (The full anatomical contour of the veneers is obtained). Exact contouring, particularly in the preparation area is recommended to avoid time consuming and risky fitting procedures.
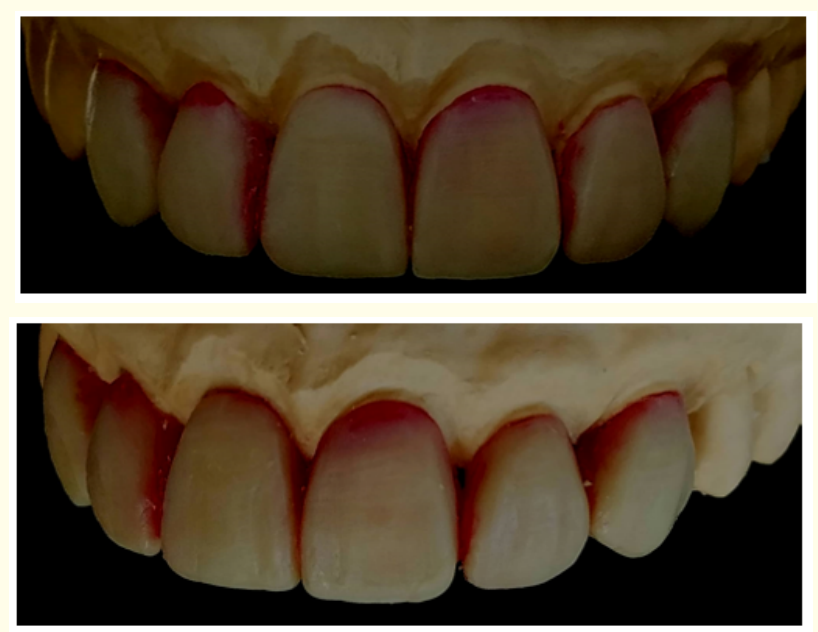

Figure 4: The waxed model.

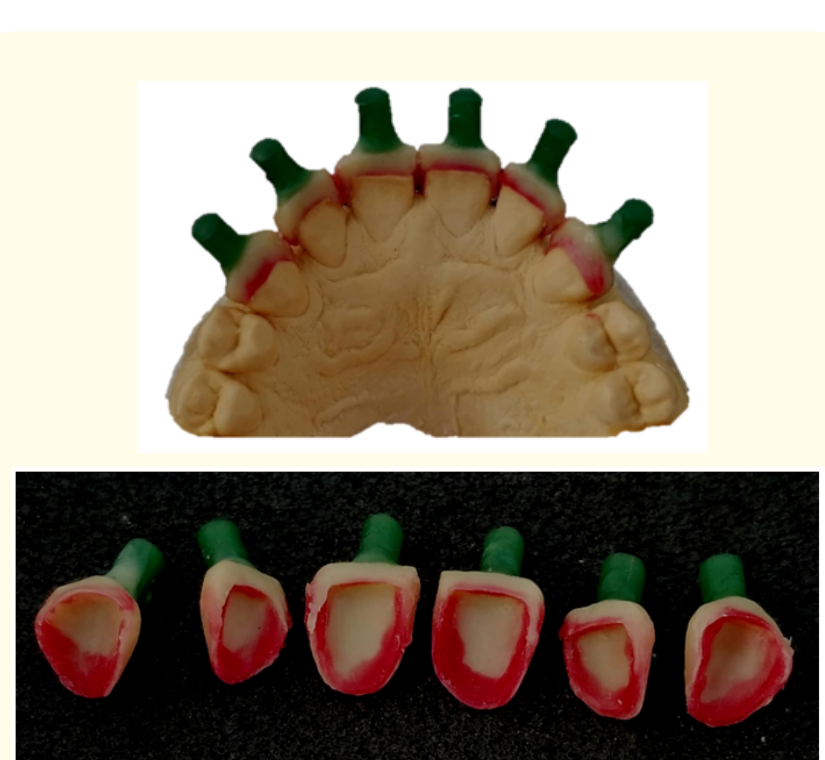

Figure 5: Sprue pins placement on wax patterns.

The wax restorations are then sprued onto the ring base former using wax. The length of the sprue pin must not exceed 5 to $6 \mathrm{~mm}$ and it depends on the ring length. The space between each pattern and the ring wall should be at least $5 \mathrm{~mm}$. $3 \mathrm{~mm}$ at least should separate the patterns and $10 \mathrm{~mm}$ of empty space should be left before the ring up opening.

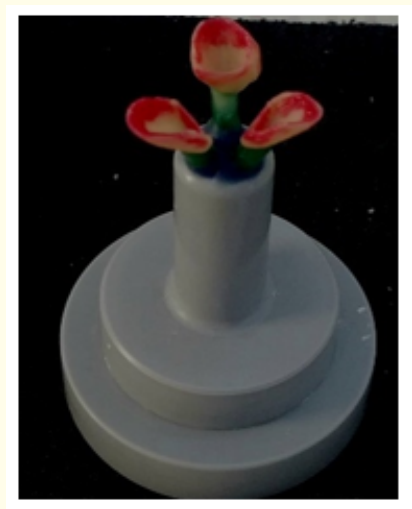

Figure 6: Sprueing the wax patterns onto the ring base. 
The next step is to proceed to the investment of the wax pattern. The investing is carried out with a specially developed carbon-free phosphate bonded speed investment for press-ceramic techniques offering an easier, faster and safer processing.

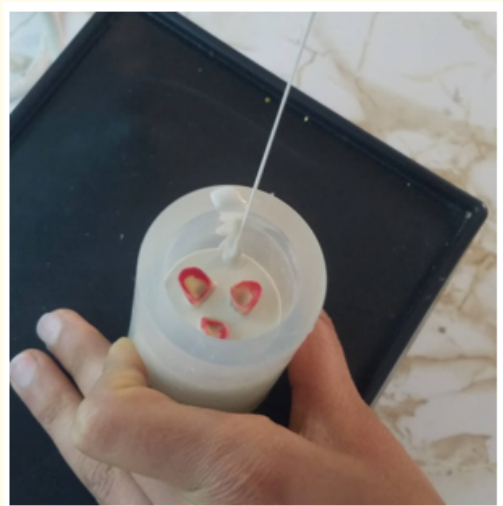

Figure 7: The investment.

The investment ring is placed with the funnel facing down in a pre-heated burn-out furnace at $850^{\circ} \mathrm{C} / 1560^{\circ} \mathrm{F}$ and keep it for minimum 45 minutes. Quick heating up at the maximum possible rate results in a relatively constant expansion of the investment, avoiding the formation of cracks and preserving the strength of the investment material.

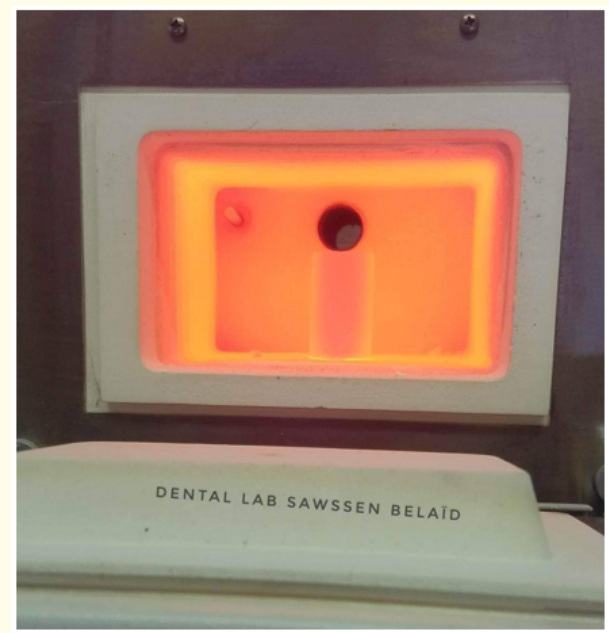

Figure 8: The investment ring preheating.
Once the preheating process is completed, the investment ring is immediately removed from the preheating furnace immediately. The pressed ceramic ingot is inserted into the investment ring with the printed side facing up. 3. A plunger is placed into the investment ring. Then the whole investment ring is placed in the center of the press furnace table. The dedicated press program is started.

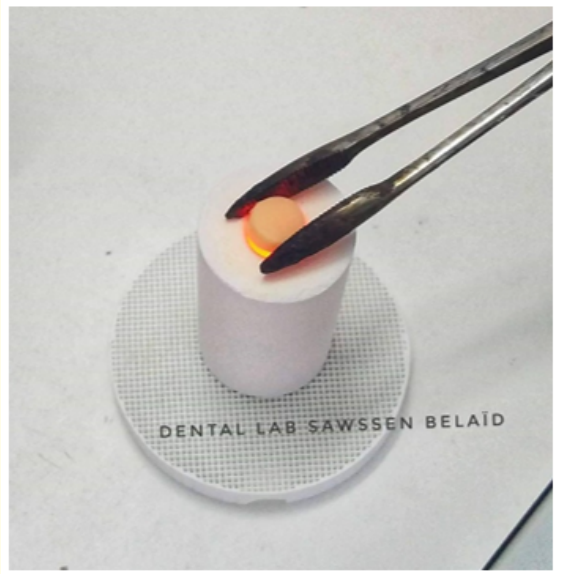

Figure 9: The ceramic ingot insertion.

After cooling, the length of plunger on the investment ring is marked. The investment ring is then cut along the marking with a suitable cut-off disk and carefully broken into two sections.

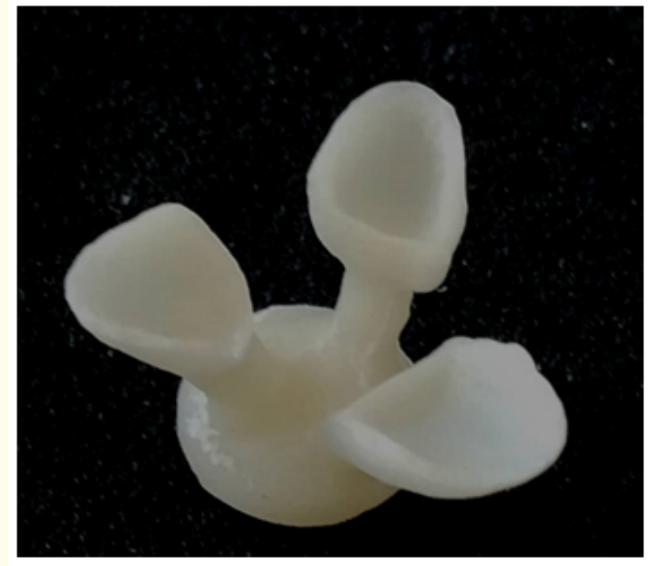

Figure 10: Casting devested. 
Rough devesting is carried out by blasting with glass beads at 4 bar pressure. Fine devesting is carried out by blasting with glass beads at 2 bar pressure. Finally the sprues are cut off with a suitable cut-off disk and the area is smoothen.

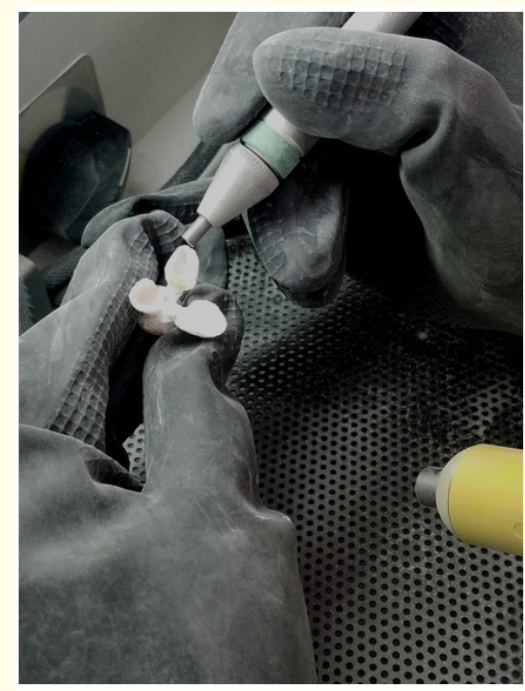

Figure 11: Devesting carrying out.

The glass-ceramic restorations are finished, using suitable grinding instrument with low speed and light pressure to prevent overheating $[9,13]$.

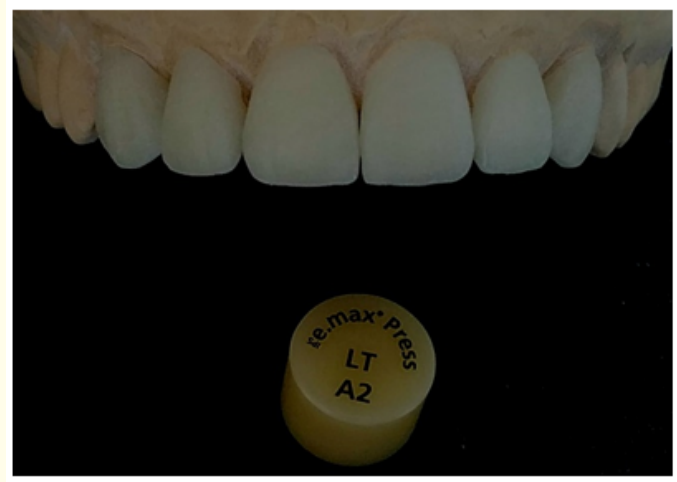

Figure 12: The ceramic veneers after finishing and polishing.

The try-in was then made. And after checking the veneers fit and the patient acceptance of the final aesthetic result, veneers were bonded.

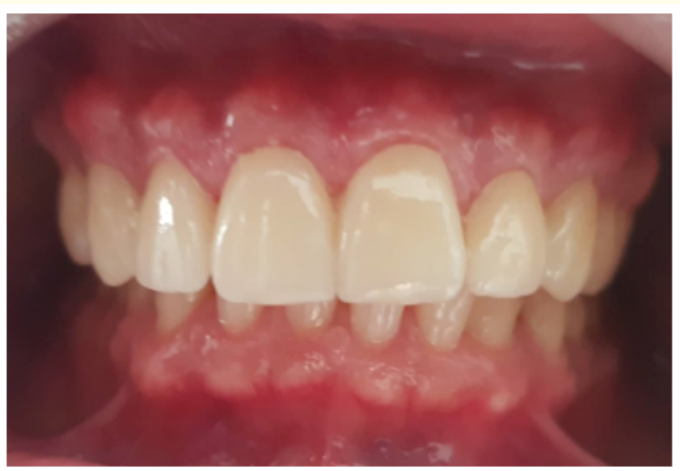

Figure 13: Intraoral view of the bonded veneers.

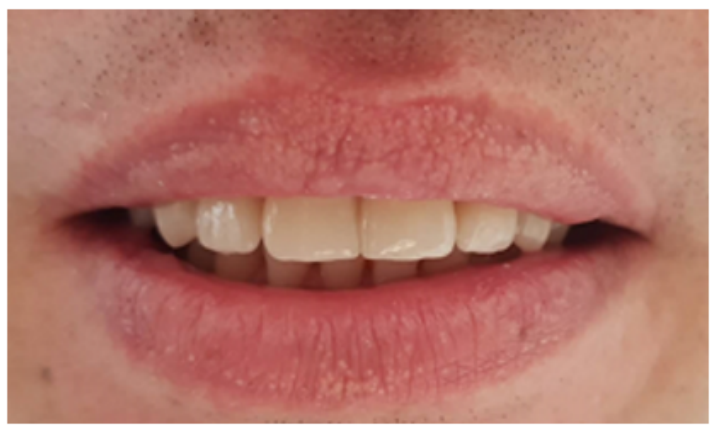

Figure 14: The final result.

\section{Discussion}

For diastema closure direct composite restorations or crowns can also be described. A minimally invasive approach should be preferred in the era of the minimally invasive dentistry. Many opinions would prefer the direct composite restorations as a faster and safer treatment option. But the longevity of these restorations id very questionable. Walls., et al. conducted a two-years clinical trial of a composite laminate veneer system for masking discoloration or hypoplasia on the anterior teeth. The technique produced an acceptable improvement in the aesthetics of the patients in the trial. However, the composite veneers were susceptible to chipping (52\% of lateral incisor) and marginal staining: $75 \%$ of veneers showed some evidence of marginal staining after two years during function3. The veneer restorations had also a deleterious effect upon the gingival health of the teeth on which they were placed. In the other hand, according to Pjetursson., et al. porcelain 
veneers show a survival rate comparable to that of metal ceramic restorations $[8,12]$.

Ceramics are considered the best in mimicking the natural tooth appearance. The optical behaviour of ceramic materials differ from system to system and this should be taken into consideration during the selection of which system to be used. The most important criteria to be considered when choosing one ceramic system upon another are translucency and resistance or mechanical behaviour. When proceeding to veneers fabrication, the aesthetic outcome is the practitioner's greatest concern. Therefore the chosen ceramic system should provide high translucency, which is the first advantage of heat pressed ceramics, especially the IPS e.max Press system. It actually offers a combination between aesthetics and strength. Added to that, this technique utilizes the experience that the lab technician already has in lost wax method with metal alloys $[1,3]$.

The choice of a glass based ceramic (lithium disilicate crystals) was also guided by the occlusal context of the patient. This reinforced ceramic could offer us besides the aesthetics, a toughness required when flexure risk factors are present. Pressed ceramic porcelains are at least two times stronger than feldspathic. The pressed ceramic restorations are far more durable and resistant to fracture. It is also less abrasive to opposing teeth than traditional feldspathic porcelain. Numerous studies show that many feldspathic materials are far more aggressive against natural dentition and causes more wear and damage [7].

However, pressed ceramics cannot be indicated in cases of severely reduced residual dentition, severely reduced occlusal vertical dimension, parafunctions (bruxism) or patients with known allergies to any of the components [10].

The respect of the different laboratory steps and the particularity of each one is necessary for a suitable result.

\section{Conclusion}

The choice of a suitable ceramic system depends on its aesthetic and mechanical criteria. To guarantee the final result the recommendations related to the used product within the different steps of the framework fabrication should be respected. The final outcome of the prosthetic project is the result of the decision made after clinical examination, the framework design [2], the choice of the framework material and also the accuracy of the laboratory steps that have been proceeded.

\section{Bibliography}

1. Amal EL Yamani. "Système Empress et restaurations prothétiques". Actualités Odonto-Stomatologiques - n² 246 (2009): 167-178.

2. CM Gormana WE. "Comparison of two heat-pressed all-ceramic dental materials”. Dental Materials 16.6 (2000) 389-395.

3. Christopher Pescatore. "The case for pressed ceramic veneers". Cosmetic dentistry and whitening (2005).

4. Gregg A Helvey. "Classification of Dental Ceramics" Inside Dentistry, Avril (2013): 62-80.

5. Jürgen Manhart. "Esthétique antérieure parfaite grâce aux facettes céramiques collées". Rev Mens Suisse Odontostomatol 121.1 (2011): 39-50.

6. Laila Al Dehailan. "Review of the Current Status of All-Ceramic Restorations". IU School of Dentistry.

7. Liliana Porojan. "Cristina Savencu Applications of Heatpressed Ceramics for Single Tooth Restorations, REV”. CHIM. (Bucharest) 67 (2016): 123-126.

8. Marcos Vargas. "A step-by-step approach to a diastema closure - a dual-purpose technique that manages black triangles, International dentistry - australasian edition 6.4 (): 32-36.

9. Master all your challenges, lisi Press.

10. Nasrin R Sadaqah. "Ceramic Laminate Veneers: Materials Advances and Selection". Open Journal of Stomatology 4.5 (2014): 268-279.

11. Núbia Pavesi Pini. "Advances in dental veneers: materials, applications, and techniques, Clinical". Cosmetic and Investigational Dentistry 4 (2012): 9-16.

12. Rakhi Gupta and Anjali Miglani. "Esthetic Management of Diastema Closure: An Innovative Technique Utilizing Putty Index Method". International Journal of Oral Care and Research 6.1(2018): 104-108.

13. Ting-Ting Tsai and Chun-Cheng Hung. "Comparison of two heat-pressed all-ceramic crown systems". The Kaohsiung Journal of Medical Sciences 20 (2004): 341-345.

\section{Volume 3 Issue 12 December 2019 (c) All rights are reserved by Zeineb Riahi., et al.}

